\title{
Risk management and operations research: a review and introduction to the special volume
}

\author{
Desheng $\mathrm{Wu}^{1}$
}

Published online: 30 December 2015

C Springer Science+Business Media New York 2015

Risks exist in all aspects of our business world. Many risks exhibit properties of interconnection and complexity besides uncertainty and dynamics, and therefore may be difficult to quantify (Arena et al. 2010, 2011; Wu and Olson 2010; Wu et al. 2014). Operations research provides many tools, such as game theory and optimization, for dealing with risks in today's uncertain world (Wu et al. 2010, 2014). Over the past several decades, risk management and operations research have attracted a great deal of attention from both researchers and practitioners, especially in the emerging area of enterprise risk management (Wu and Olson 2009, 2010, 2013). Figure 1 presents the numbers of journal publications on "risk management and operations research" since 1994. The trend figure was created by using the key words "risk management and operations research" in ISI Web of Science (SCI-EXPANDED). Data we used from ISI Web of Science include the data source Science Citation Index Expanded (SCI-EXPANDED). Figure 1 suggests that risk management and operations research have been popularized by the financial crisis during the last two decades and continue to be hot research areas.

We are very pleased to offer this special volume on state-of-the-art research and development of quantitative analysis in the areas of risk management and operations research.

Deutsch and Golany analyze an inspection game between a single inspector and several independent (potential) violators over a finite-time horizon. In each period, the inspector gets a renewable inspection resource, which cannot be saved and used in future periods. The authors introduce an efficient way to determine a Nash equilibrium for this game, parametrically in the inspector's global budget. The results indicate that there are situations where the players choose the same strategy even when the values of beta are changed. The authors also conduct an interesting review of business intelligence and risk management, and present relations between them and game theory.

Hausken and Zhuang analyze the tradeoff between safety and production. The government chooses safety effort and tax rate in the first stage, and then the company strikes a balance between safety effort and production in the second stage. Both players' safety efforts mitigate

Desheng Wu

dwu@ucas.ac.cn

1 School of Management, University of Chinese Academy of Sciences, Beijing, China 


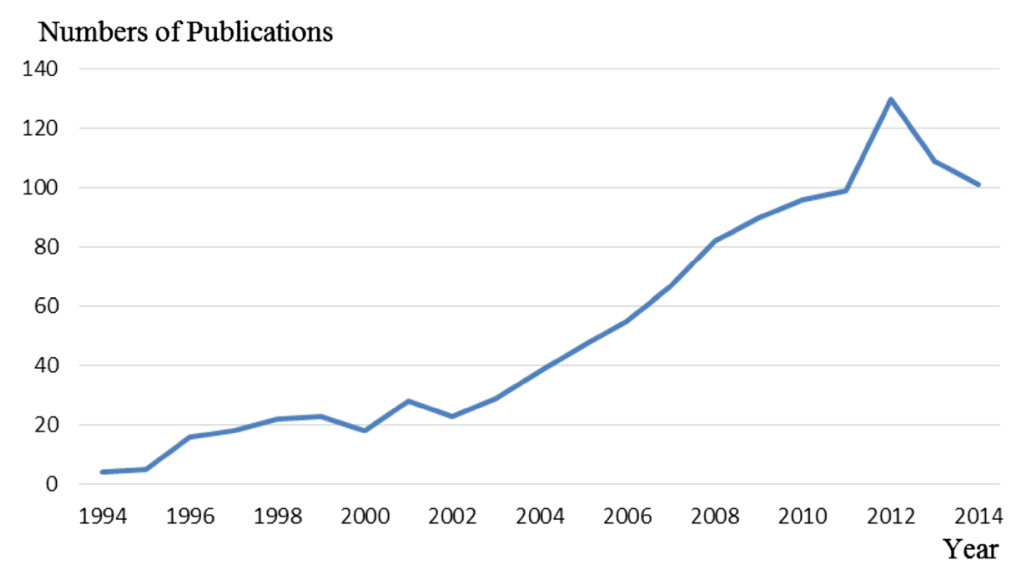

Fig. 1 Publication trends on "risk management and operations research" since 1994

the negative impact of a disaster. The authors model the disaster probability as a contest between the disaster magnitude and the two players' safety efforts. They derive several implications from the model. First, as the safety effort of one player approaches infinity, the marginal change in the other player's safety effort, with respect to the first player's safety effort, approaches to zero. Second, an infinitely large safety effort by any player causes the disaster probability and the negative impact of the disaster to decrease toward a constant. Third, as one player's safety effort approaches infinity, the other player's safety effort approaches zero. Fourth, the two players' safety efforts are strategic substitutes so that an increase in one player's safety effort decreases the other player's safety effort. This enables the players to free ride on each other's safety efforts. Fifth, a higher tax rate causes the company to exert a higher safety effort. Sixth, with a maximum tax rate the company focuses exclusively on safety effort and generates no profit. Seventh, the company's safety effort is inverse $\mathrm{U}$ shaped in the disaster magnitude.

Kwon and Li et al.'s work considers bounds for the price of a European-style call option under regime switching. Stochastic semi-definite programming models are developed that incorporate a lattice generated by a finite-state Markov chain regime-switchingmodel as a representation of scenarios (uncertainty) to compute bounds. The optimal first-stage bound value is equivalent to a Value at Risk quantity, and the optimal solution can be obtained via simple sorting. The upper (lower) bounds from the stochastic model are bounded below (above) by the corresponding deterministic bounds and are always less conservative than their robust optimization ( $\min -\max$ ) counterparts. The authors demonstrate the value of the stochastic solution (bound) and computational experiments using the S\&P 500 index that illustrating the advantages of the stochastic programming approach over the deterministic strategy.

Choi explores the multi-period risk minimization inventory models for fashion product purchasing by use of a mean-variance approach. The author finds that the optimal ordering quantity is increasing in the expected profit target, decreasing in the number of periods of the season, and increasing in the market interest rate. The author also proposes and solves several extended models that consider realistic and timely industrial measures such as minimum ordering quantity, carbon emission tax, and carbon quota. The author analytically derives the necessary and sufficient condition(s) for the existence of the optimal solution for each model and shows how the purchasing budget, the profit target, and the market interest rate affect the optimal solution. 
Moazeni, Coleman, and Li investigate an approach based on smoothing and parametric rules to minimize mean and conditional value-at-risk (CVaR) of the execution cost using Monte Carlo simulations. The proposed approach reduces computational complexity by smoothing the nondifferentiability arising from the simulation discretization and by employing a parametric representation of a stochastic strategy. The authors further handle constraints using a smoothed exact penalty function. Using the downside risk as an example, they show that the proposed approach can be generalized to other risk measures. In addition, they computationally illustrate the effect of including risk in the stochastic optimal execution strategy.

Rodríguez-Puerta and Álvarez-López consider a model of production allocation in the context of the theory of the firm under uncertainty. This is the case of a firm that has just produced a known amount of an output and can allocate it to two possible ends: one with a certain price, the other with an uncertain price. The authors first establish conditions to determine whether the firm will make use of both ends or of only one of them. They then find a limit value for the frontier price below which the firm will decide to allocate the total amount of production to the uncertain end. They finally analyze an application concerning the middleman who buys the firm's output in the certain end. In two specific cases, the authors also provide closed-form expressions for the optimal allocation to both ends and for the frontier price.

Sun, Wissel, and Jackson analyze financial hedging tools for inventory management in a risk-averse corporation. They consider the problem of optimizing simultaneously over both the operational policy and the hedging policy of the corporation in a multiproduct model. They demonstrate that the contribution is a separation result such that for a corporation with multiple products and inventory departments, the inventory decisions of each department can be made independently of the other departments' decisions. That is, no interaction needs to be considered among different products. The authors formulate the combined inventory management and financial hedging problem, and show how standard results enable the problem to be separated into independent single-item problems. The separation result can be generalized to a multiperiod setting, and solved via dynamic programming techniques.

Javanmardi and Lawryshyn introduce a new rank dependent utility approach, which unlike existing models, provides an SSD efficient portfolio as a function of the investors' quantified risk aversion degrees. A parametric family of distortion functions is considered to model various levels of risk aversion. Under assumptions of equally probable scenarios, for any distortion function the corresponding optimization models can be expressed as a linear program and easily solved. An empirical study is performed to compare the performance of the proposed model to the existing portfolio selection models in the literature.

Cong and Tan analyze the value-at- risk (VaR) based optimal risk management solution using reinsurance under a class of premium principles that is monotonic and piecewise. The monotonic piecewise premium principles include not only those that preserve stoploss ordering, but also the piecewise premium principles that are monotonic. By adopting the monotonic piecewise premium principle, the proposed optimal reinsurance model has a number of advantages. It has the flexibility of allowing the reinsurer to use different risk loading factors for a given premium principle or to use entirely different premium principles depending on the layers of risk. It can also analyze the optimal reinsurance strategy in the context of multiple reinsurers that may use different premium principles (as attributed to the difference in risk attitude and/or imperfect information). Furthermore, by artfully imposing certain constraints on the ceded loss functions, the resulting model can be used to capture the reinsurer's willingness and/or capacity to accept risk or to control counterparty risk from the perspective of the insurer. Under some technical assumptions, the authors derive explicitly the optimal form of the reinsurance strategies in all the above cases. They show that a truncated 
stop-loss reinsurance treaty or a limited stop-loss reinsurance treaty can be optimal depending on the constraint imposed on the retained and/or ceded loss functions.

Romanko and Mausser use conditional value-at-risk (CVaR) - based proxies for VaR objectives based on a heuristic algorithm for scenario-based value-at-risk (VaR) optimization. The heuristic algorithm obtains robust results with low computational complexity, which solves the problem of high computational complexity in VaR optimization.

Krzemienowski and Szymczyk present a copula-based extension of conditional valueat-risk and its application to portfolio optimization. Copula-based conditional value-at-risk $(\mathrm{CCVaR})$ is a scalar risk measure for multivariate risks modeled by multivariate random variables. It is assumed that the univariate risk components are perfect substitutes, i.e., they are expressed in the same units. CCVaR is a quantile risk measure that allows one to emphasize the consequences of more pessimistic scenarios. By changing the level of a quantile, the measure permits one to parameterize prudent attitudes toward risk ranging from the extreme risk aversion to the risk neutrality. This difference between CCVaR and CVaR allows one to efficiently solve CCVaR portfolio optimization problems based on the full information carried by a multivariate random variable by employing column generation algorithm.

Ray and Jenamani propose two models for optimal order allocation in a newsvendor setting, where both supply and demand are uncertain. The first model considers a risk neutral decision maker who maximizes the total expected profit under disruption risk. The second one is for a risk-averse decision maker who does so under service level constraints. Analytical closed form solutions for both models are derived. To overcome the computational complexity of the exact optimal solution, two algorithms are developed to generate optimal order quantity and the corresponding set of suppliers. The solutions with exact optimization algorithms and the proposed ones are illustrated and compared with numerical examples. The results show that the proposed algorithms give the exact optimal solution while being tractable. Finally, a case study is used to illustrate the applicability of the proposed model.

Gaffney and Ben-Israel study an insurance model, with realistic assumptions about coverage, deductible, and premium. Insurance is shown to decrease the variance of the cost to the insured, but increase the expected cost, a trade-off that places the proposed model in the Markowitz mean-variance model.

Choi et al. conduct a comprehensive review that identifies pioneer work and pioneer scientists in enterprise risk management using data in both Scopus and ISI Web of Science. The authors also conduct a literature review of the best-ranked scholars' papers and critical analysis of the work of Wu and Olson, especially from an enterprise risk management perspective, to glean implications and suggestions for the optimization and customization of enterprise risk management.

Acknowledgments This work is partially supported by National Natural Science Foundation of China (NSFC) Grant (Grants \# 71471055, 91546102), the 1000-Talents plan Program for the Young Scientists. We thank all the referees and the Editor-in-Chief for many valuable suggestions, their energy, and effort in bringing out this special volume.

\section{References}

Arena, M., Arnaboldi, M., \& Azzone, G. (2010). The organizational dynamics of enterprise risk management. Accounting Organizations and Society, 35(7), 659-675.

Arena, M., Arnaboldi, M., \& Azzone, G. (2011). Is enterprise risk management real? Journal of Risk Research, 14(7), 779-797. 
Wu, D. D., \& Olson, D. L. (2009). Enterprise risk management: Small business scorecard analysis. Production Planning \& Control, 20(4), 362-369.

Wu, D. D., \& Olson, D. L. (2010). Introduction to special section on risk and technology. Technological Forecasting and Social Change, 77(6), 837-839.

Wu, D. D., Xie, K., Chen, G., \& Gui, P. (2010). A risk analysis model in concurrent engineering product development. Risk Analysis, 30(9), 1440-1453.

Wu, D. D., Zheng, L., \& Olson, D. L. (2014). A decision support approach for online stock forum sentiment analysis. IEEE Transactions on Systems Man and Cybernetics, 44(8), 1077-1087.

Wu, D. S. D., \& Olson, D. L. (2013). Computational simulation and risk analysis: An introduction of state of the art research Preface. Mathematical and Computer Modelling, 58(9-10), 1581-1587. 